\title{
Evaluation of germline RET proto-oncogene variants in Peruvian patients with medullary thyroid carcinoma
}

\author{
Claudia Barletta-Carrillo ${ }^{1,2,3 *}$, Sandro Casavilca-Zambrano ${ }^{3}, M^{a}$ del Carmen Castro-Mujica ${ }^{4}$, and \\ Julio A. Poterico ${ }^{5}$
}

${ }^{1}$ Genetics Service, Instituto Nacional de Enfermedades Neoplásicas; ${ }^{2}$ Human Genetics Laboratory, Faculty of Biological Sciences, Universidad Nacional Mayor de San Marcos; ${ }^{3}$ Department of Pathology, Instituto Nacional de Enfermedades Neoplásicas; ${ }^{4}$ School of Human Medicine, Universidad Ricardo Palma; ${ }^{5}$ Genetics Service, Instituto Nacional de Salud del Niño-San Borja. Lima, Perú

\begin{abstract}
Background: About $25 \%$ of patients suffering from medullary thyroid carcinoma (MTC) have been associated to germinal pathogenic variants of RET proto-oncogene. Multiple endocrine neoplasia type 2 (MEN2) and familial medullary thyroid carcinoma (FMTC) are phenotypes related germinal RET pathogenic alterations. Despite the vast research on genomics about MTC in European descendants, little is known in Latin America. Objective: The aim of this study was to assess germinal pathogenic genetic variants of RET proto-oncogene in a Peruvian cohort of patients with MTC. Materials and Methods: We conducted a descriptive, observational, and retrospective study of patients with diagnosis of MTC who attended at least one genetic consultation at a national Peruvian oncologic healthcare institute, whose germinal genetic results of RET gene were available on clinical records. Genetic analysis was carried out using the Sanger sequencing methodology evaluating RET gene exons: 10,11,13,14,15, and 16. We collected personal and familial information and morphological tumor-relevant information. Results: We found $28.6 \%$ (6/21) of probands diagnosed with MTC carrying a germinal pathogenic variant of RET gene. Half or these germline alterations were de novo. We identified five germinal pathogenic variants: p.Cys620Ser, p.Cys630Ser, p.Cys634Gly, p.Cys634Arg, and p.Leu790Phe. Conclusion: This is the first report of germinal pathogenic variants of RET proto-oncogene found in Peruvian patients with MTC, with unique findings highlighting the importance of genomic analysis for precise diagnosis for personalized clinical management.
\end{abstract}

Key words: Thyroid cancer. Medullary. Multiple endocrine neoplasia type 2. Familial medullary thyroid carcinoma. RET Proto-Oncogene. Genetic testing. Genotype-Phenotype correlation.

\section{Introduction}

Medullary thyroid carcinoma (MTC) represents a rare tumor of $\mathrm{C}$ parafollicular cells, accounting $5-10 \%$ of thyroid malignancies, and being responsible $\sim 13.4 \%$ of thyroid cancer-associated deaths $\mathbf{1}^{1,2}$. One quarter of patients with MTC could pertain to the group of hereditary cancer syndromes, with patients showing multifocal, bilateral, and other manifestations ${ }^{3,4}$. Germinal pathogenic variants of $R E T$ proto-oncogene (Rearranged during
Transfection) have been associated with genetics disorders, such as multiple endocrine neoplasia type 2 (MEN2), Hirschsprung disease (HSCR), and familial MTC (FMTC) ${ }^{3}$. MTC has a penetrance of $80-100 \%$ in MEN2 and CMTF and due to an autosomal dominant inheritance pattern, having a 50\% chance of inheriting the risk of this malignancy to the offspring ${ }^{5}$.

The RET proto-oncogene contains 21 exons, located on long arm of chromosome 10 (locus 10q11.2);

\section{Correspondence:}

${ }^{*}$ Claudia Barletta-Carrillo

E-mail: claudiabarletta@ @otmail.com
Date of reception: $24-03-2020$

Date of acceptance: 07-05-2020

DOI: 10.24875/HGMX.20000050
Available online: 12-11-2020

Rev Med Hosp Gen Mex. 2020;83(4):159-167 www.hospitalgeneral.mx NC-ND license (http://creativecommons.org/licenses/by-nc-nd/4.0/) 
codifying for a tyrosine kinase receptor (protein RET). This protein is mainly expressed in precursor cells of the neural crest and urogenital tract, acting as receptor for ligands such as glial cell-derived neurotrophic factor (GDNF) family. RET protein activation takes place in normal conditions by a complex of coreceptors and ligands that include two groups of proteins: (1) the GNDF family of ligands (GFLs) such as neurturin, artemin, and persephin and (2) the glycosylphosphatidylinositol-anchored GDNF-family $\alpha$ receptors (GFR $\alpha$ s) ${ }^{3,6}$.

$R E T$ is a one-step transmembrane protein, composed of three functional domains: the extracellular ligand-binding region, the transmembrane portion, and the cytoplasmic tyrosine kinase domain. Four cadherin-like and cysteine-rich yuxtamembrane regions conform the extracellular domain. On the other hand, the intracellular domain contains two tyrosine kinase subdomains (TK1 and TK2) which participate in several transduction pathways activation involved in cell survival, proliferation, differentiation, migration, and chemotaxis ${ }^{6,7}$.

Phenotype correlated to RET proto-oncogene variants seem to depend on codon change rather than type of amino acid substitution. Variants reported in MEN2A and FMTC affect primarily the extracellular cysteine-rich domain, and less frequently the tyrosine kinase domain ${ }^{8}$. In MEN2A, most germinal RET gene alterations distort codon 634 in around $85 \%$ of cases, with Cys634Arg substitution as the most common. On the other hand, FMTC correlates to pathogenic variants on RET-exons 5, 8, 10, 11, 13, 14, 15, and 16. Conversely, MEN2B has been associated to germinal pathogenic changes of the tyrosine kinase subdomain 2, with $95 \%$ of patients showing alteration in codon 918 , and $5 \%$ in codon $883^{4,6,9-12}$.

Confirmation of germinal pathogenic variants of RET proto-oncogene can help starting a personalized diagnosis and management and could be a good preventive measure to reduce mortality in non-affected relatives carriers of germinal genetic pathogenic alterations ${ }^{13}$. Moreover, Latin American populations lack of extensive personalized genetic and molecular tests, conversely to European descendants, limiting genotype-phenotype comparability across diverse populations ${ }^{14}$.

For this reason, we started - for the $1^{\text {st }}$ time in Peru - in our institution the implementation of molecular analysis of this key proto-oncogene in a subset of Peruvian patients with diagnosis of MTC and suspicion of a hereditary cancer syndrome (MEN2 or FMTC). We identified genetic variants and show the genotype-phenotype correlation in this case series.

\section{Materials and methods}

\section{Patients characteristics}

We included 21 unrelated consecutive probands with incoming diagnosis of MTC. These patients had at least one evaluation by a medical geneticist in the outpatient consultation or during hospitalization. We collected clinical, surgical, and pathological reports of our case indexes or - if possible - their relatives. For pedigree's elaboration, we follow the standardized nomenclature suggested by the National Society of Genetic Counselors ${ }^{15}$. Each consultation and evaluation by a clinical geneticist provided to patients and their relatives a detailed information about the molecular test for RET gene. In so doing, the blood withdrawal was obtained after proband's verbal or signed informed consent. Our project was reviewed and approved by the Institution Review Board (IRB) of Instituto Nacional de Enfermedades Neoplasicas (INEN), with IRB code INEN17-68.

\section{Genomic analysis}

Genomic DNA was extracted from periferic blood collected into EDTA tubes, using the High Pure polymerase chain reaction (PCR) Template Kit (Roche Diagnostics $\mathrm{GmbH}$, Mannheim, Germany), according to manufacturer's specifications. DNA was quantified with Qubit $^{\mathrm{TM}}$ Fluorometer (Invitrogen ${ }^{\mathrm{TM}}$, Carlsbad, CA, USA). A priori selected exons of the RET proto-oncogene were amplified using the PCR technique. Final volume in these experiments was $25 \mu \mathrm{L}$, containing: $80-100 \mathrm{ng}$ of DNA template, $0.4 \mu \mathrm{M}$ of both primers, PCR Buffer $1 \times, 3-4 \mathrm{mM}$ of $\mathrm{Mg}^{2+}$, and $0.625 \mathrm{U}$ of the enzyme Platinum Taq DNA Polymerase High Fidelity (Invitrogen). $\mathrm{Mg}^{2+}$, dNTPs, and DNA template concentrations were standardized for each exon independently. We used the Veriti thermocycler (LifeTechnologies), with specific primers for exons and exon-intron limit ( 25 bp in-deep intronic position $)^{16}$. The PCR thermal conditions started with a pre-heating cycle at $95^{\circ} \mathrm{C}$ for $3 \mathrm{~min}$ followed by 35 cycles of denaturation step at $95^{\circ} \mathrm{C}$ for $30 \mathrm{~s}$, annealing at $60-62^{\circ} \mathrm{C}$ for $30 \mathrm{~s}$, and extension at $68^{\circ} \mathrm{C}$ for $40 \mathrm{~s}$, and a final extension cycle at $68^{\circ} \mathrm{C}$ for $7 \mathrm{~min}$.

Bands of PCR products were observed in a $3 \%$ agarose gel carried out at $100 \mathrm{~V}$ for $45 \mathrm{~min}$, dyed with SYBR $^{\circledR}$ Safe for 15 min. PCR products were purified with the PureLink ${ }^{\mathrm{TM}}$ Quick PCR Purification Kit (Invitrogen); quantifying amplicons with fluorometry using Qubit $^{\mathrm{TM}}$ Fluorometer (Invitrogen ${ }^{\mathrm{TM}}$, Carlsbad, CA, USA). 
Bidirectional resequencing was carried out with BigDye Terminator version 3.1 Cycle Sequencing Kit and the ABI PRISM 3500 Genetic Analyzer (Applied Biosystems $^{\circledR}$, Foster City, CA, USA), using same exon-specific primers as aforementioned and 12-14 ng of final purified amplicon. DNA sequence analyses were performed with SeqScape ${ }^{\circledR}$ v3.0 (Life Technologies).

Variants were referred to cDNA sequence of RET with accession number NM_020975.5, GRCh38.p27. We described variants following nomenclature guidelines of the Human Genome Variation Society (HGVS) site: http://www.hgvs.org/content/guidelines. ClinVar (URL: $\quad$ https://www.ncbi.nlm.nih.gov/clinvar/?ter$\mathrm{m}=\mathrm{RET} \% 5$ Bgene $\% 5 \mathrm{D}$ ) and ARUP (University of Utah, URL: http://www.arup.utah.edu/database/) databases were revised for searching of germinal RET variants published elsewhere.

\section{Results}

From a total of 21 patients (11 women, 10 men) in our case series, we report main clinical and histologic findings, for example, age of MTC diagnosis and RET proto-oncogene analysis results (Table 1). A positive family history of MTC or PTC was found in three (Prob 4, 6, and 17) of our patients, whose RET molecular analysis showed a germinal pathogenic variant (Figs. 1-2). Our group of patients has been diagnosed at a median age of 37 years old (IQR: 32-49), with the youngest aged 7 years old. Three of our patients (Prob 1, 11, and 14) suffered of a mixed thyroid carcinoma (MTC and PTC) synchronically. One of them (Prob 11) shows a RET gene analysis with a variant of unknown significant (VUS) and a concomitant germinal pathogenic variant (c.2370 G > T; p.Leu790Phe) (Fig. 3).

Special phenotypic findings were observed in some patients: hepatobiliary neoplasm in proband 13, parathyroid adenoma in proband 7, hyperparathyroidism (HPT), and pheochromocytoma (Pheo) in proband 17. The two latter patients have MEN2A confirmed diagnosis. On the other hand, none of our patients show until date any MEN2B clinical manifestation (i.e., mouth neuromas, marfanoid habitus, and intestinal ganglioneuromatosis).

This cohort shows a characteristic clinical profile according to available data on clinical records. For instance, 8 of 19 patients had bilateral thyroid disease, with most of them presenting metastasis at diagnosis. Thyroidectomy was performed in $90.6 \%$ (19/21) of our index cases, being the radical or bilobulated extirpation procedures the preferred for surgeons. The remaining two patients presented inoperable thyroid cancer.
Six of our probands (28.6\%) with MTC had a germinal RET pathogenic variant (Prob 4, 6, 11, 13, 17 y 18) identified with Sanger resequencing, confirming the diagnosis of predisposition cancer syndrome related to MTC. Half of these patients (Prob 4, 6 y 17) reported a positive family history of cancer. Furthermore, we found five - in our six index cases - different missense pathogenic variants of the RET proto-oncogene exons: p.Cys620Ser (exon 10), p.Cys630Ser, p.Cys634Gly, p.Cys634Arg (both in exon 11), and p.Leu790Phe (exon 13) (Figs. 1-6). Detected pathogenic variants in the cystein-rich yuxtamembrane domain of the RET protein were found in $83.3 \%(5 / 6)$ of our positive cases - these mutations affect cystein residues of extracellular domain. Most of the genetic changes were attributed to exon $11(66.6 \%)$, and half of our patients with a germinal RET proto-oncogene alteration depict a harmful change at codon position 634 of RET protein.

Additional genetic findings help us to better describe our case series. In Proband 11, we detected a concomitant - to the pathogenic variant p.Leu790Phe - VUS on exon 13: c.2371T > C, producing a change on codon 791 (p.Tyr791His). Moreover, benign variants were observed in our patients, such as p.Gly691Ser, p.Leu769Leu, and p.Ser904Ser, with frequencies of $81.0 \%$ (17/21), 95.2\% (20/21), and 81.0\% (17/21), respectively. Most of these nucleotidic changes were found in homozygosis. On the other hand, one of our 21 patients depicted a benign variant (c.2608-24G > A) on intron 14 .

\section{Discussion}

This report demonstrates high frequency $(28.6 \%)$ of germinal pathogenic variants in Peruvian patients with MTC and suspicion of MEN2 or FMTC. This finding agrees with the observation that $25 \%$ of patients with MTC would carry a pathogenic change on the proto-oncogene $R E T^{1,4,5}$. Moreover, most of our cases with MEN2 or FMTC molecularly corroborated were above 30 years old, differing from other report where patients aged $\leq 30$ years depicted higher rates - compared to older than 30 - of germinal pathogenic variants $(50 \%$ vs. $13.5 \%$, respectively) ${ }^{17}$.

Germinal variants of RET proto-oncogene associated to MEN2 or FMTC occur due to changes in exon-codificating the cystein-rich and the activable intracellular tyrosine kinase domains of this transmembrane protein ${ }^{6}$. Phenotypic expression of MEN2 or FMTC seems to correlates with specific changes on RET protein codons $^{10}$; hence, clinical recommendations depend on type of genetic alteration reported ${ }^{18,19}$. 


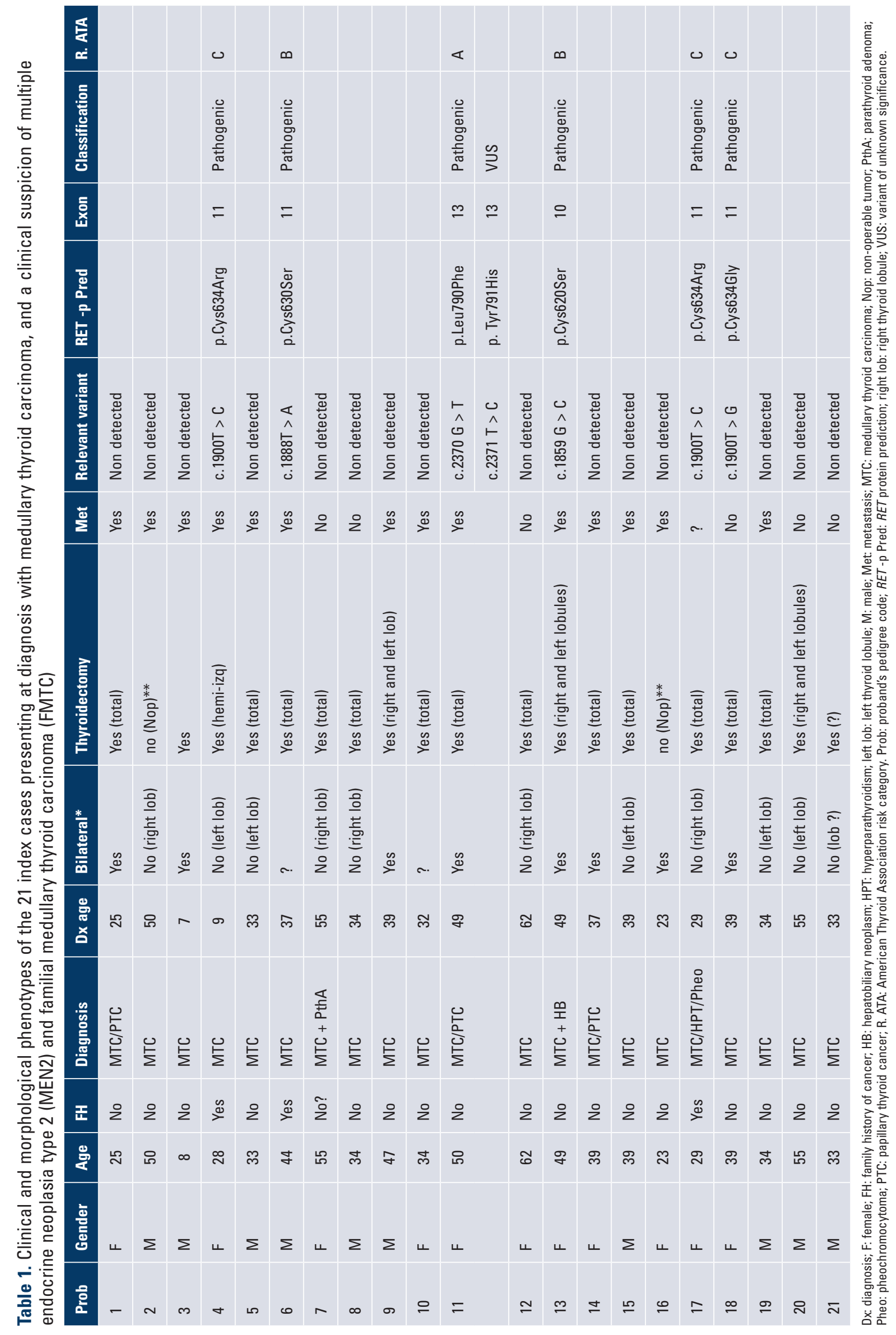


A Prob-04

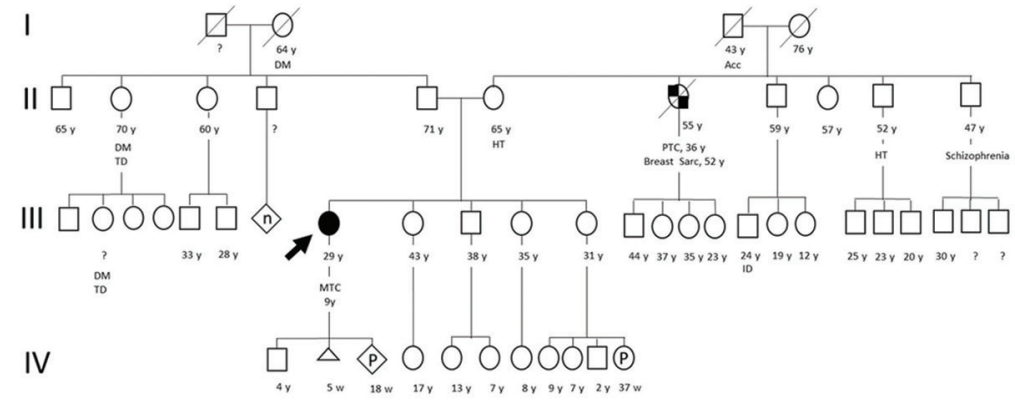

B

CGACGAGCTGYGCCGCACGGT

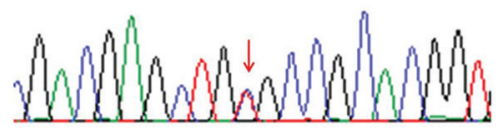

Figure 1. A: pedigree of proband 4. B: electropherogram of proband 4: pathogenic variant c.1900T > C (p.Cys634Arg). Acc: accident; DM: diabetes mellitus; HT: hypertension; ID: intellectual disability; MTC: medullary thyroid carcinoma; PTC: papilar thyroid cancer; Sarc: sarcoma; TD: non-specified thyroid disease.

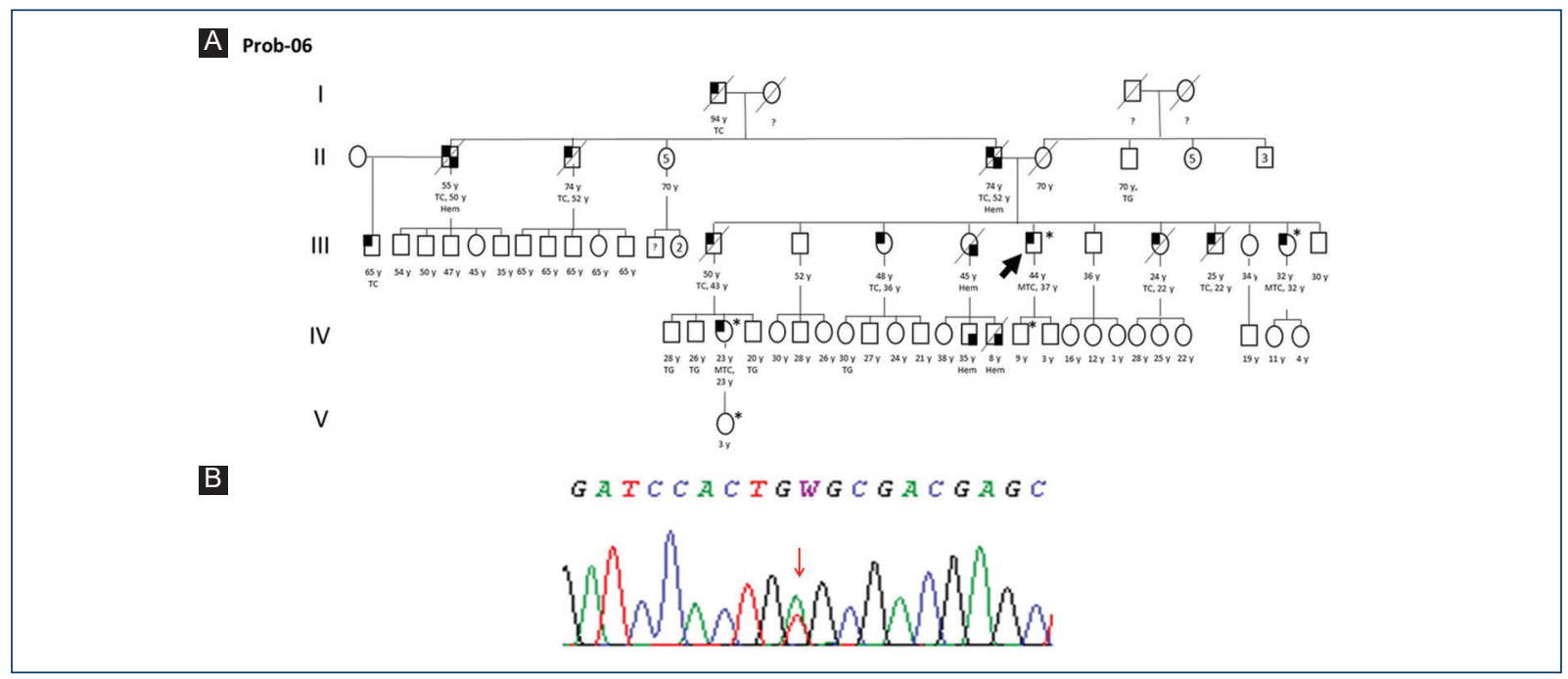

Figure 2. A: pedigree of proband 6. MTC: medullary thyroid carcinoma, TC: thyroid carcinoma, TG: thyroid goit, Hem: hemophilia. B: electropherogram of proband 6: pathogenic variant c.1888T > A (p.Cys630Ser).

Codon 634 of RET protein seems to be affected in $85 \%{ }^{6}$ of cases associated with MEN2A mainly due to a substitution of cystein by arginine (c.1900T > C; p.Cys634Arg), resulting a relevant predictor of Pheo and parathyroid disease ${ }^{20,21}$. The proband 17 had a diagnosis of MEN2A due to MTC, Pheo, and HPT, presenting the variant c.1900T > C of RET gene, with a strong family history: 2 first-degree, 1 second-degree, and 2 third-degree relatives with MTC or unspecified thyroid carcinoma (Fig. 5). According to ATA 4 , this index case pertains to group $C$ risk classification, the highest risk group related to MEN2A and FMTC.

Tyrosine specific residues of RET protein which is activated through phosphorilation can help secondary tranducers to continue the intracellular signaling. To date, up to 18 phosphorylation sites have been reported, for example, codon 791 (Tyr791) $^{6,22}$. One of our patients (Prob 11) carries a pathogenic variant of $R E T$ 


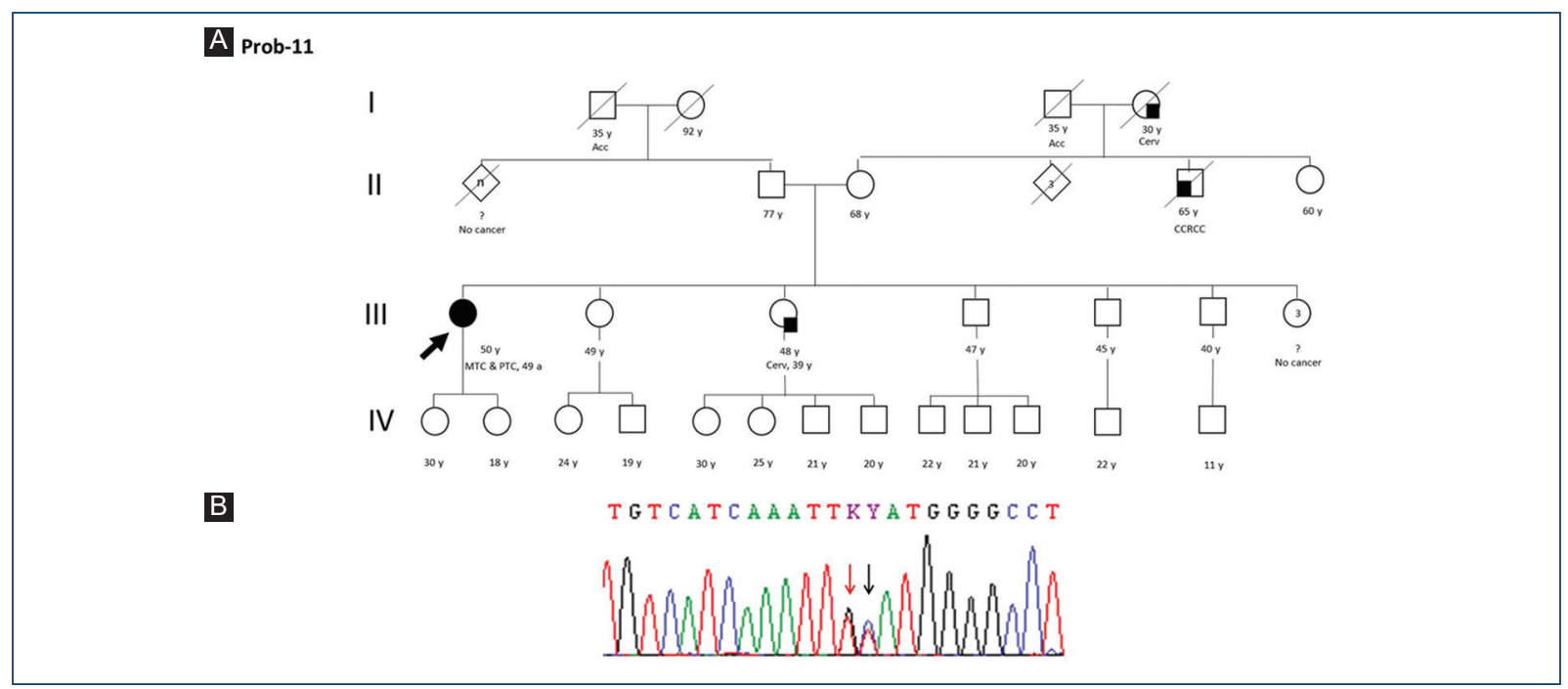

Figure 3. A: pedigree of proband 11. B: electropherogram of proband 11: pathogenic variant c.2370 G > T(p. Leu790Phe) marked with red arrow and a VUS c.2371 T > C (p. Tyr791His) marked with black arrow.

Acc: accident; CCRCC: clear cell renal cell carcinoma; Cerv: cervix cancer; MTC: medullary thyroid carcinoma; PTC: papilar thyroid cancer.

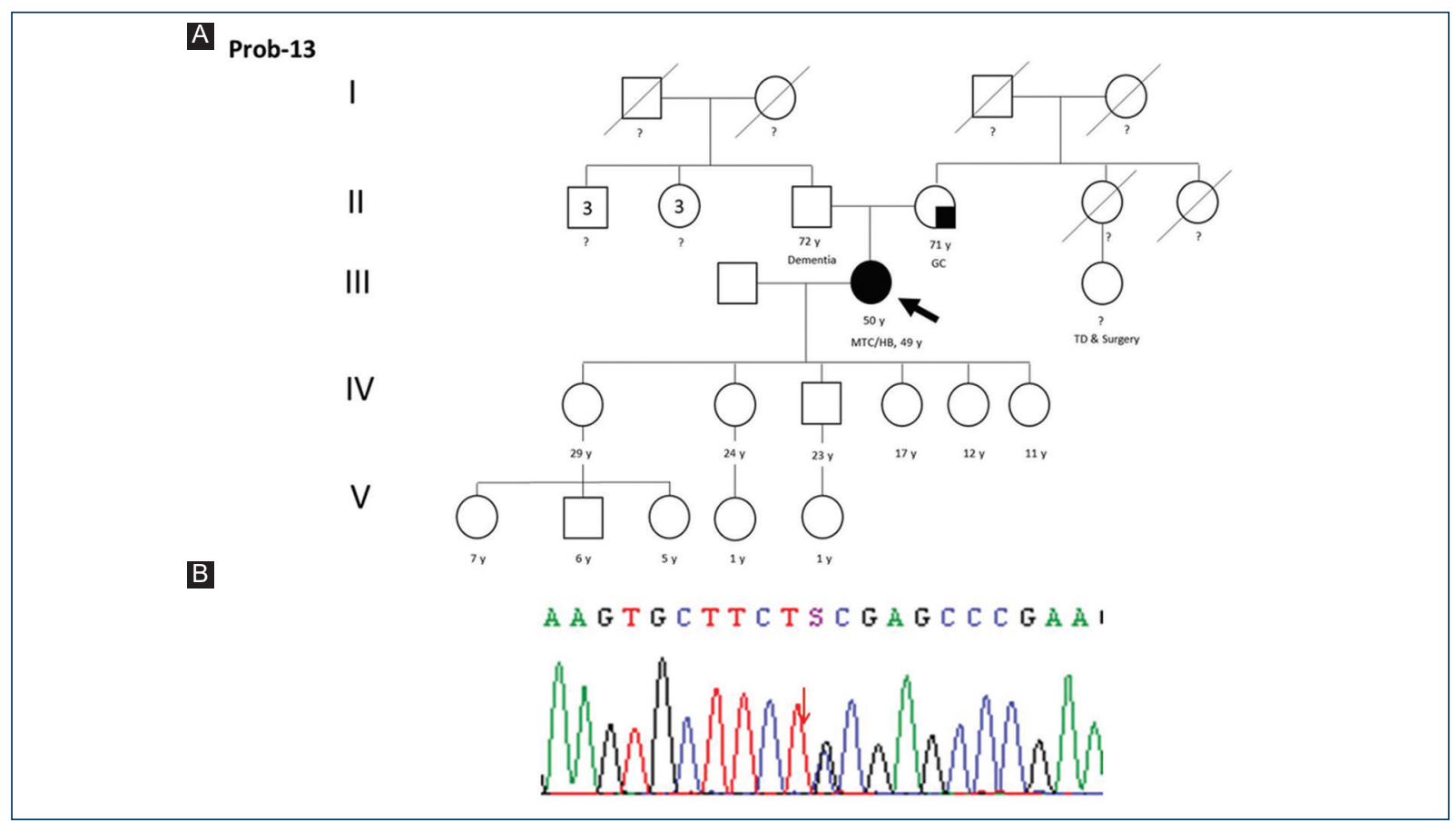

Figure 4. A: pedigree of proband 13. MTC: medullary thyroid carcinoma, GC: gastric cancer, TD: non-specified thyroid disease, HB: hepatobiliary neoplasm. B: electropherogram of proband 13: pathogenic variant C.1859 G > C (p.Cys620Ser).

proto-oncogene: c.2370 G > T (p.Leu790Phe) and a VUS c.2371 T > C (p.Tyr791His). Histologic findings in this female patient demonstrated a mixed thyroid carcinoma (MTC and PTC), without oncologic family history.
The germinal pathogenic change corresponds to category A following 2009 ATA guideline ${ }^{4}$. Additional aminoacid substitutions in codon 791, as p.Tyr791Asn and Tyr791Phe, are found in ClinVar named as VUS or 


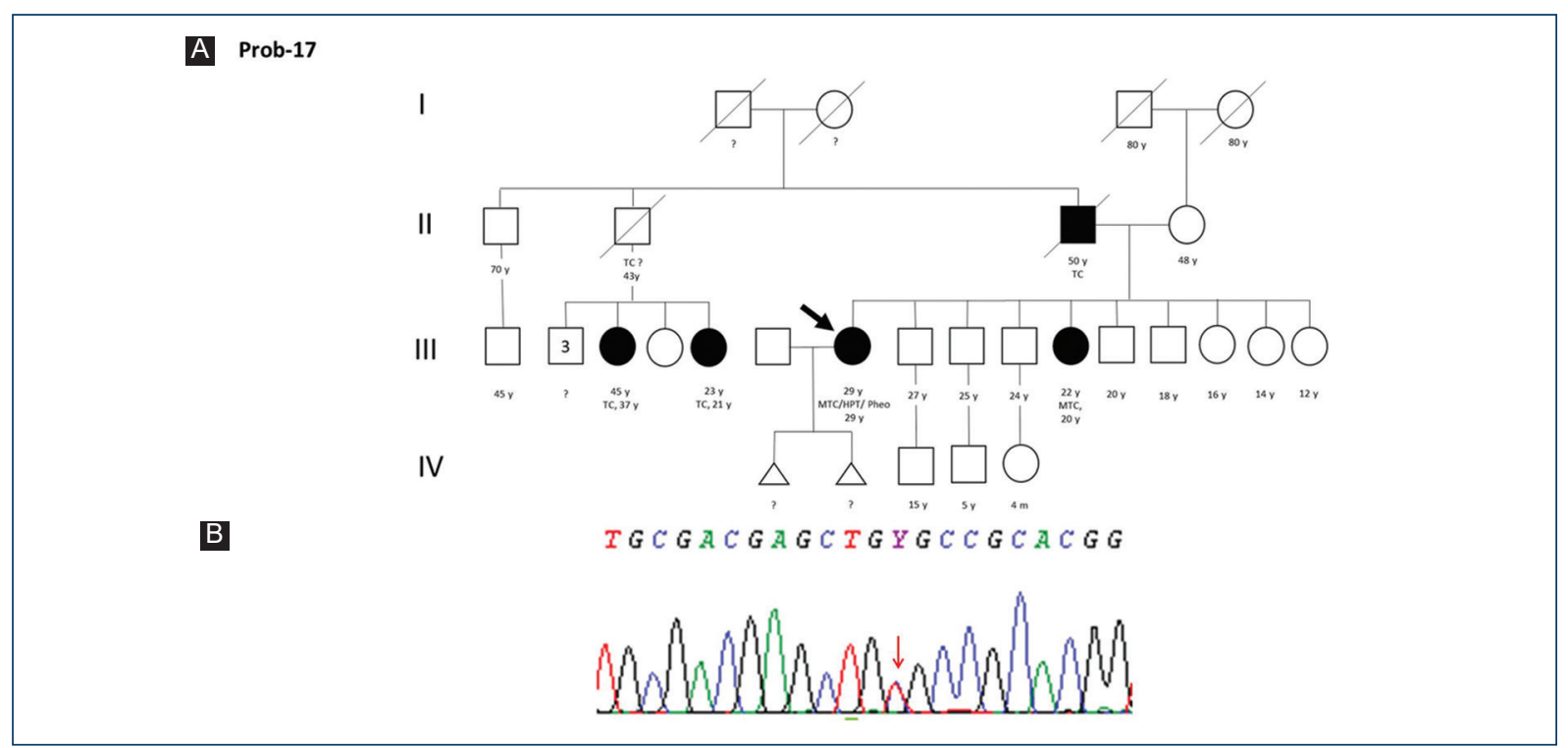

Figure 5. A: proband 17. MTC: medullary thyroid carcinoma, TC: thyroid carcinoma, HPT: hyperparathyroidism, Pheo=pheochromocytoma. B: electropherogram of proband 17: pathogenic variant c.1900T > C (p.Cys634Arg).

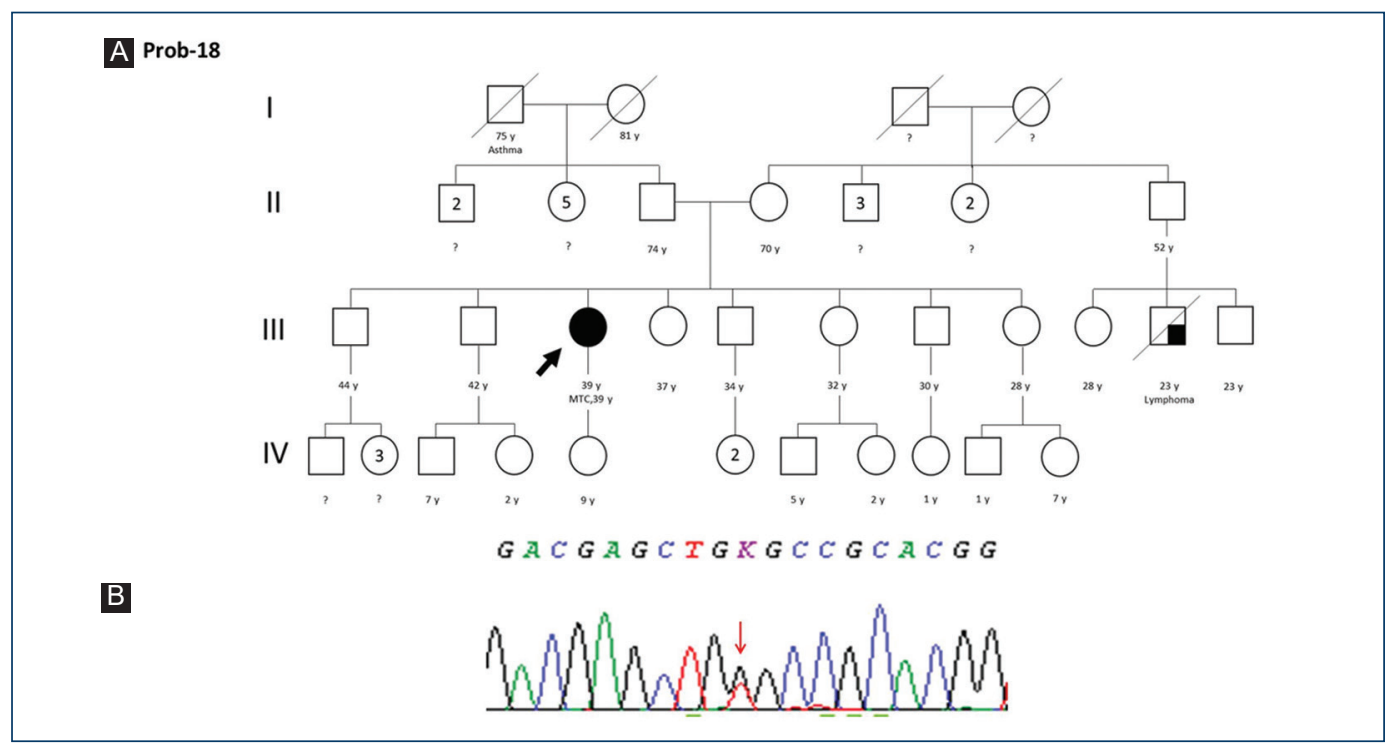

Figure 6. A: proband 18. MTC: medullary thyroid carcinoma. B: electropherogram of proband 18: pathogenic variant c. $1900 \mathrm{~T}>\mathrm{G}$ (p.Cys634Gly).

with conflicting interpretations of pathogenicity, respectively. The latter also belongs to ATA risk group A, therefore a minor-risk category.

Another relevant genetic change on RET protein has been reported for RET c.1888T > A (p.Cys630Ser) detected in proband 6 . This male patient of 44 years old had a strong famility history of thyroid carcinoma: 6 of first-degree, 4 of second-degree, and one of third-degree relatives. We were able to perform germinal molecular analysis of RET proto-oncogene in five relatives. Thus, two MTC-affected relatives (III-23 and IV-3) carry the p.Cys630Ser variant, likewise in two apparently disease-free, first- (IV-15) and third-degree (V-1) relatives - aged 9 and 3 years, respectively (Fig. 2). This variant correspond to ATA risk category $B$, with prophylactic thyroidectomy recommendation before 5 years old. Although this is a known mutation spot ${ }^{23-26}$, our detected genetic alteration with a amino acid 
replace of cysteine by serine, is unique to the best of our knowledge, and this could suggest a Peruvian genetic alteration transmitted trough generation in the Andean region. Further, epidemiological studies would help to confirm this hypothesis.

Ten to fifteen percent of people with diagnosis of MEN2A or FMTC show alteration in codons $609,611,618$, or 620 in exon $10^{5}$. However, Hansen et al. ${ }^{27}$ suggest that exon 10 alteration of RET protein could be more common than previously stated in FMTC. They reported mutations on this exon in 8 of 10 evaluated families. Proband 13 carries a pathogenic variant in codon 620 (p.Cys620Ser) (Fig. 4). She depicts an unusual and ever reported (to our knowledge) metachronic presentation of MTC and hepatic metastasic cholangiocarcinoma.

Asymptomatic patients carrying a germinal pathogenic variant of $R E T$ proto-oncogene would benefit from prophylactic thyroidectomy, despite serum calcitonin lev$\mathrm{els}^{28}$. Other studies support this surgical approach at appropriate age for disease-free survival outcomes ${ }^{29-32}$.

We can find current risk classification categories, proposed by health-care institutions through genotype-phenotype correlations. For instance, ATA shows four categories from $A$ to $D$, according to the risk associated of developing an aggressive MTC at young $\mathrm{age}^{4}$. Thus, the Category $\mathrm{D}$ suggests the highest risk, recommending prophylactic thyroidectomy in the $1^{\text {st }}$ year of life. Categories $B$ and $C$ suggest this surgical procedure before 5 years old and at older ages if the asymptomatic patients is ranked in the Category $\mathrm{A}$.

In 2010, The North American Neuroendocrine Tumor Society consensus guideline for the diagnosis and management of neuroendocrine tumors published recommendations classifying variants of the RET proto-oncogene in three groups, as follows: level 1 , low risk (codons 609, 768, 790, 791, 804, and 891), level two, high risk (codons 611, 618, 620, and 634), and level 3 , the highest risk (codons 883, 918, and 922). Recommended age for prophylactic thyroidectomy for these risk groups is as follows: around 5 years old (but $<10$ if not performed before), before 5 years old and within the first 6 months of life, respectively ${ }^{33}$.

This report represents the first case series of Peruvian patients with diagnosis of MTC and with molecular confirmation of germinal RET proto-oncogene variants. The patients were derived to a national neoplastic institute. Moreover, we analyzed relevant exons associated to MEN2 or FMTC, focusing in a priori exons-selected analysis. Nevertheless, most pathogenic variants reported in the literature in patients with these diseases located at RET exons included in our work.
On the other hand, one limitation of this report was the difficulty of evaluating punctual pathogenic variants in all proband's relatives. However, we demonstrated in one family (Prob 6) the importance of performing this personalized genetic test for preventive action.

This is the first report of germinal pathogenic variants of RET proto-oncogene found in Peruvian patients with MTC, with some unique findings, demonstrating the importance of molecular analysis for corroborating clinical diagnosis, starting adequate prevention, and including personalized management in countries with limited resources.

\section{Conclusion}

We demonstrated a high frequency of pathogenic germline variants of the RET proto-oncogene, half of them being de novo. Furthermore, we found a peculiar genetic alteration in a large family with several affected members, which could be unique to the Peruvian population and could have a founder effect. This manuscript reinforces the importance of developing genomic and personalized medicine in the Peruvian health system.

\section{Acknowledgments}

We thank to all patients and their relatives who collaborated by giving us their information and clinical information. Moreover, we are grateful to all our professors and colleagues from the Genetics Service of the National Institute of Neoplastic Diseases (INEN).

\section{Conflicts of interest}

The authors have no conflicts of interest to declare.

\section{Ethical disclosures}

Protection of human and animal subjects. The authors declare that no experiments were performed on humans or animals for this study.

Confidentiality of data. The authors declare that they have followed the protocols of their work center on the publication of patient data.

Right to privacy and informed consent. The authors declare that no patient data appear in this article.

\section{References}

1. Leboulleux S, Baudin E, Travagli JP, Schlumberger M. Medullary thyroid carcinoma. Clin Endocrinol. 2004;61:299-310. 


\section{Barletta-Carrillo, et al: RET proto-oncogene in Peruvians}

2. Xu L, Wang WB, Zhao YP, Zhang TP, Liao Q, Chen G, et al. Medullary thyroid carcinoma with nodular goiter carries an excellent prognosis. J Surg Oncol. 2012;106:169-73.

3. Online Mendelian Inheritance in Man. Available from: https://www.omim. org. [Last accessed on 2018 Feb 28]

4. American Thyroid Association Guidelines Task Force, Kloos RT, Eng C Evans DB, Francis GL, Gagel RF, Gharib H, et al. Medullary thyroid cancer: management guidelines of the American thyroid association. Thyroid. 2009;19:565-612.

5. Raue F, Rondot S, Schulze E, Szpak-Ulczok S, Jarzab B, Frank-Raue K Clinical utility gene card for: multiple endocrine neoplasia Type 2. Eur J Hum Genet EJHG. 2012;20:142.

6. de Groot JW, Links TP, Plukker JT, Lips CJ, Hofstra RM. RET as a diagnostic and therapeutic target in sporadic and hereditary endocrine tumors. Endocr Rev. 2006;27:535-60.

7. Krampitz GW, Norton JA. RET gene mutations (genotype and phenotype) of multiple endocrine neoplasia Type 2 and familial medullary thyroid carcinoma. Cancer. 2014:120:1920-31.

8. Eng C, Clayton D, Schuffenecker I, Lenoir G, Cote G, Gagel RF, et al The relationship between specific RET proto-oncogene mutations and disease phenotype in multiple endocrine neoplasia Type 2. International RET mutation consortium analysis. JAMA. 1996;276:1575-9.

9. Carlson KM, Dou S, Chi D, Scavarda N, Toshima K, Jackson CE, et al Single missense mutation in the tyrosine kinase catalytic domain of the RET protooncogene is associated with multiple endocrine neoplasia Type 2B. Proc Natl Acad Sci U S A. 1994;91:1579-83.

10. Eng C, Smith DP, Mulligan LM, Nagai MA, Healey CS, Ponder MA et al. Point mutation within the tyrosine kinase domain of the RET proto-oncogene in multiple endocrine neoplasia Type $2 \mathrm{~B}$ and related sporadic tumours. Hum Mol Genet. 1994;3:237-41.

11. Smith DP, Houghton C, Ponder BA. Germline mutation of RET codon 883 in two cases of de novo MEN 2B. Oncogene. 1997;15:1213-7.

12. Gimm O, Marsh DJ, Andrew SD, Frilling A, Dahia PL, Mulligan LM, et al. Germline dinucleotide mutation in codon 883 of the RET proto-oncogene in multiple endocrine neoplasia Type 2B without codon 918 mutation. J Clin Endocrinol Metab. 1997;82:3902-4.

13. Jindrichová S, Vcelák J, Vlcek P, Neradilová M, Nemec J, Bendlová B. Screening of six risk exons of the RET proto-oncogene in families with medullary thyroid carcinoma in the Czech republic. J Endocrinol. 2004; 183:257-65.

14. Bustamante CD, Burchard EG, De la Vega FM. Genomics for the world Nature. 2011:475:163-5.

15. Bennett RL, French KS, Resta RG, Doyle DL. Standardized human pedigree nomenclature: update and assessment of the recommendations of the national society of genetic counselors. J Genet Couns. 2008;17:424-33.

16. Alvandi E, Akrami SM, Chiani M, Hedayati M, Nayer BN, Tehrani MR et al. Molecular analysis of the RET proto-oncogene key exons in patients with medullary thyroid carcinoma: a comprehensive study of the Iranian population. Thyroid. 2011;21:373-82.

17. Kihara M, Miyauchi A, Yoshioka K, Oda H, Nakayama A, Sasai $H$, et al Germline RET mutation carriers in Japanese patients with apparently sporadic medullary thyroid carcinoma: a single institution experience. Auris Nasus Larynx. 2016:43:551-5.

18. Wells SA, Asa SL, Dralle H, Elisei R, Evans DB, Gagel RF, et al. Revised American thyroid association guidelines for the management of medullary thyroid carcinoma. Thyroid. 2015;25:567-610.
19. Elisei R, Alevizaki M, Conte-Devolx B, Frank-Raue K, Leite V, Williams GR. 2012 European thyroid association guidelines for genetic testing and its clinical consequences in medullary thyroid cancer. Eur Thyroid J. 2013:1:216-31.

20. Mulligan LM, Eng C, Healey CS, Clayton D, Kwok JB, Gardner E, et al. Specific mutations of the RET proto-oncogene are related to disease phenotype in MEN 2A and FMTC. Nat Genet. 1994;6:70-4.

21. Schuffenecker I, Billaud M, Calender A, Chambe B, Ginet N, Calmettes C, et al. RET proto-oncogene mutations in French MEN 2A and FMTC families. Hum Mol Genet. 1994;3:1939-43.

22. Liu X, Vega QC, Decker RA, Pandey A, Worby CA, Dixon JE. Oncogenic RET receptors display different autophosphorylation sites and substrate binding specificities. J Biol Chem. 1996;271:5309-12.

23. Kitamura Y, Goodfellow PJ, Shimizu K, Nagahama M, Ito K, Kitagawa W, et al. Novel germline RET proto-oncogene mutations associated with medullary thyroid carcinoma (MTC): mutation analysis in Japanese patients with MTC. Oncogene. 1997;14:3103-6.

24. Maciel RM, Camacho CP, Assumpção LV, Bufalo NE, Carvalho AL, de Carvalho GA, et al. Genotype and phenotype landscape of MEN2 in 554 medullary thyroid cancer patients: the BrasMEN study. Endocr Connect. 2019;8:289-98.

25. González B, Salcedo M, Medrano ME, Mantilla A, Quiñónez G, Benítez-Bribiesca L, et al. RET oncogene mutations in medullary thyroid carcinoma in Mexican families. Arch Med Res. 2003;34:41-9.

26. Elisei R, Romei C, Cosci B, Agate L, Bottici V, Molinaro E, et al. RET genetic screening in patients with medullary thyroid cancer and their relatives: experience with 807 individuals at one center. J Clin Endocrinol Metab. 2007;92:4725-9.

27. Hansen HS, Torring $H$, Godballe $C$, Jäger AC, Nielsen FC. Is thyroidectomy necessary in RET mutations carriers of the familial medullary thyroid carcinoma syndrome? Cancer. 2000;89:863-7.

28. Wells SA, Chi DD, Toshima K, Dehner LP, Coffin CM, Dowton SB, et al. Predictive DNA testing and prophylactic thyroidectomy in patients at risk for multiple endocrine neoplasia Type 2A. Ann Surg. 1994;220: 237-47.

29. Machens A, Niccoli-Sire P, Hoegel J, Frank-Raue K, van Vroonhoven TJ, Roeher HD, et al. Early malignant progression of hereditary medullary thyroid cancer. N Engl J Med. 2003;349:1517-25.

30. Skinner MA, Moley JA, Dilley WG, Owzar K, Debenedetti MK, Wells SA. Prophylactic thyroidectomy in multiple endocrine neoplasia Type 2A. N Engl J Med. 2005;353:1105-13.

31. Schreinemakers JM, Vriens MR, Valk GD, de Groot JW, Plukker JT, Bax KM, et al. Factors predicting outcome of total thyroidectomy in young patients with multiple endocrine neoplasia Type 2: a nationwide long-term follow-up study. World J Surg. 2010;34:852-60.

32. Sanso GE, Domene HM, Garcia R, Pusiol E, de M, Roque M, et al. Very early detection of RET proto-oncogene mutation is crucial for preventive thyroidectomy in multiple endocrine neoplasia Type 2 children: presence of C-cell malignant disease in asymptomatic carriers. Cancer. 2002;94:323-30

33. Chen H, Sippel RS, O'Dorisio MS, Vinik Al, Lloyd RV, Pacak K, et al. The North American neuroendocrine tumor society consensus guideline for the diagnosis and management of neuroendocrine tumors: pheochromocytoma, paraganglioma, and medullary thyroid cancer. Pancreas. 2010;39:775-83. 